\title{
Filigrane
}

Écoutes psychanalytiques

\section{L'influence de la théorie du Moi-peau sur le travail avec les tout-petits}

\section{Christine Anzieu-Premmereur}

Volume 27, numéro 1, 2018

La transmission de la psychanalyse

URI : https://id.erudit.org/iderudit/1055600ar

DOI : https://doi.org/10.7202/1055600ar

Aller au sommaire du numéro

Éditeur(s)

Revue Santé mentale au Québec

ISSN

1192-1412 (imprimé)

1911-4656 (numérique)

Découvrir la revue

Citer cet article

Anzieu-Premmereur, C. (2018). L'influence de la théorie du Moi-peau sur le travail avec les tout-petits. Filigrane, 27(1), 17-29.

https://doi.org/10.7202/1055600ar
Résumé de l'article

Le Moi-peau est une théorie selon laquelle contenir et permettre la continuité et l'intégration sont des priorités quand on traite enfants et patients fragiles, de façon à maintenir une expérience de sécurité du Moi-peau, condition première pour se sentir vivant et croître. Les thérapies conjointes parents-enfants permettent d'intervenir à la fois au niveau intrasubjectif et interpersonnel. Un rôle essentiel de l'analyste est d'aider les parents à créer un espace transitionnel. Le jeu est un élément essentiel de cette technique, en donnant aux parents l'accès au jeu avec leur enfant qui fait alors des expériences qui peuvent modifier sa relation avec ses objets internes. 


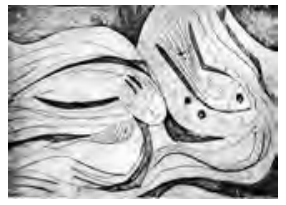

\title{
L'influence de la théorie du Moi-peau sur le travail avec les tout-petits ${ }^{1}$
}

\section{Christine Anzieu-Premmereur}

\begin{abstract}
Résumé: Le Moi-peau est une théorie selon laquelle contenir et permettre la continuité et l'intégration sont des priorités quand on traite enfants et patients fragiles, de façon à maintenir une expérience de sécurité du Moi-peau, condition première pour se sentir vivant et croître. Les thérapies conjointes parents-enfants permettent d'intervenir à la fois au niveau intrasubjectif et interpersonnel. Un rôle essentiel de l'analyste est d'aider les parents à créer un espace transitionnel. Le jeu est un élément essentiel de cette technique, en donnant aux parents l'accès au jeu avec leur enfant qui fait alors des expériences qui peuvent modifier sa relation avec ses objets internes.
\end{abstract}

Mots clés: dépression primaire; espace transitionnel; jeu; Moi-peau; enveloppes psychiques; thérapie mère-enfant.

Abstract: The Skin Ego theory demonstrates that containment, continuity and integration are priorities when working with children and vulnerable patients, in order to maintain a secure experience of the skin ego, which is the primary condition to feel alive and to thrive. Dyadic therapy helps to provide intervention at intrasubjective and interpersonal levels. The analyst's role is to help parents create a transitional space. Playing is essential to give parents access to play with their baby, who then can experience new relations with internal objects.

Key Words: mother-child psychotherapy; primary depression; play; psychic enveloppes; skin ego; transitional space.

e suis une psychiatre française formée à la Société psychanalytique de Paris à l'époque des débats entre André Green et Jean Laplanche sur la pulsion, et de la remise du corps et de l'affect au centre de la pensée analytique, longtemps réduite au niveau du langage par Lacan.

Je suis devenue analyste d'enfants dans les années 80 avec des supervisions en analyse d'adulte avec Jean-Luc Donnet et en analyse d'enfants avec Serge Lebovici et René Diatkine, quand j'étais responsable d'un département de psychiatrie infantile au Centre Alfred Binet, un lieu hautement analytique consacré à l'enfance. C'est là que j'ai créé avec des collègues le 
Centre pour parents et nourrissons, commençant un travail que je n'ai plus jamais arrêté et qui me passionne.

J'ai aussi la chance très œedipienne d'être la fille de deux psychanalystes, Annie et Didier Anzieu, longtemps sources d'inhibition et d'idéalisation.

J'ai développé une façon d'intervenir dans les consultations et thérapies parents-bébés, avec une pensée analytique fondée sur la métapsychologie freudienne, l'aspect économique interne à l'appareil psychique mis en évidence par l'école psychosomatique de Paris, la liberté de jouer que donne Winnicott et la théorie du Moi-Peau.

\section{Le Moi-peau}

L'approche théorique de Didier Anzieu pendant les années 70 a mis l'accent sur le corps et le rôle des sensations précoces, associant sans préjugé la théorie des pulsions et celle de l'attachement. Le contact de la peau, le toucher maternel associé au holding et les expériences précoces sont décrites dans leur rôle fondamental pour le développement du moi et du fonctionnement inconscient à l'âge adulte, avec les troubles narcissiques associés à cette période primaire. Associant la sensualité avec les pulsions, D. Anzieu met en valeur le rôle primordial de la capacité de contenir les émotions, précédant celui de former des fantasmes. Les contenants deviennent alors les éléments essentiels pour le maintien d'une capacité interne organisant la capacité fantasmatique: les notions d'enveloppe psychique et de signifiant formel sont la suite de la théorie du Moi-peau.

Cette métaphore de la peau sert à représenter l'enveloppe psychique, introjectée à partir de la préoccupation maternelle, qui poursuit la protection utérine par le travail psychique d'une continuité amoureuse; le Moipeau fait référence à l'internalisation d'une capacité de ressentir, contenir et transformer sensations et émotions en représentations et affects, plus tard en pensées. C'est une interface qui lie sentir qu'on touche, être touché et se toucher soi-même. Le contact avec le corps maternel à l'occasion des soins et de la tendresse donne le sens d'une peau continue et commune avec la mère.

C'est une protection contre les stimulations externes, un filtre à fonction économique, c'est-à-dire de régulation des quantités ressenties, tandis que la partie interne de cette enveloppe enregistre et garde en mémoire ce qui a été ressenti, plaisir comme douleur. C'est à la fois une enveloppe d'excitation et de communication, gestion de la quantité et de la qualité des expériences.

Une séparation prématurée de la peau commune avec la mère donne lieu dans l'après-coup à des présentations de corps abîmé, déchiré, écorché. 
L'image mentale que l'enfant crée à partir des sensations s'associe avec le sentiment de croissance du self, d'un Moi capable de contenir la vie psychique. Le fantasme de peau commune peut disparaître alors sans traumatisme, et le narcissisme est appuyé sur cette expérience.

L'équilibre économique entre les tensions internes et l'excitation libidinale est maintenu par cette capacité contenante. On est proche de ce que Damasio écrivait il y a quelques années sur le fond continu de sensations qui est fait des sensations corporelles indifférenciées et sur lequel s'adosse l'appareil psychique s'il peut contenir l'excitation associée.

Tout commence avec le corps maternel; son odeur, sa qualité de toucher, la voix et les rythmes, intégrées comme parties du self. Puis les parties détachables du corps vont être les précurseurs des objets transitionnels; les cheveux, les lunettes, les bijoux, les vêtements, les foulards. Ces expériences répétées confirment un sens de continuité. Cette sécurité fonde le narcissisme primaire.

Ainsi, le corps musculaire s'associe aux sensations viscérales et la surface sensible de la peau peut contenir les stimulations des zones érogènes.

Le Moi-peau a plusieurs fonctions:

- maintenir la capacité de penser;

- contenir affects et émotions;

- protéger des débordements et impacts traumatiques;

- faire fonctionner l'inter-sensorialité;

- offrir une recharge libidinale et être le support de l'excitation sexuelle;

- garder en mémoire les expériences;

- et permettre l'individuation.

Quand ces fonctions sont en place, le Moi pensant se développe.

Un support physique et psychique solide intégré comme une partie du corps propre par l'enfant provient de la qualité du holding maternel et devient ce que J. Grotstein a appelé l'«objet d'arrière-plan » de l'identification primaire, à savoir un soutien du dos physique et mental. Les tout-petits enfoncés dans la dépression primaire manifestent ce besoin en tombant en arrière, ou en cherchant un appui du dos.

Un enfant de 15 mois au balancement compulsif dont la mère était envahie d'affects dépressifs a pu réintégrer un plaisir auto-érotique quand ses parents ont commencé à caresser son dos et ses cheveux avec tendresse.

C'est en observant la pathologie que l'on découvre combien le sens de soi-même est associé au rôle du corps. Frances Tustin a décrit les enfants 
autistes de la façon suivante: «Sans peau ni corps, mais avec une armure qui prétend défendre contre la terreur de se dissoudre et tomber. C'est dans la thérapie qu'on les voit acquérir un corps solide avec une structure interne.»

Bob est un nourrisson de 3 mois dont la mère s'est dissociée dans une rupture psychotique après la naissance. Elle peut être douce et proche et soudain devenir persécutée et intrusive. Bob la regarde, ébauche un sourire, puis hurle et se désorganise, le corps éclaté avec les membres éparpillés. Quand une infirmière s'occupe de lui après qu'il ait été séparé de sa mère, il cesse de sourire, refuse les biberons et son corps est couvert d'eczéma. Réunis à nouveau dans une unité spéciale de l'hôpital psychiatrique, mère et fils se touchent, se regardent; l'eczéma disparait, après une période où le bébé s'est raidi dans un mouvement de retrait avant de se laisser aller dans le sein maternel.

La fonction de miroir de la mère rapportée par Winnicott n'est pas seulement une expérience visuelle: c'est à travers toutes les modalités sensorielles que la dyade communique et que la mère renvoie à l'enfant la qualité de ce qu'il ressent. Les défauts de cette fonction entrainent une multitude d'enjeux narcissiques.

Paul est un petit de 15 mois, mal équilibré dans sa marche, confus et déconnecté du monde s'il est éloigné du corps de sa mère. Si elle ne le touche plus, il n'échange alors plus de regards ni de sons. Or, elle est trop déprimée pour faire attention à lui. C'est quand j'ai associé à ma parole le toucher caressant de son dos qu'il a pu se rassembler et se mettre à marcher pour aller chercher un jouet, et ce n'est qu'en appuyant son dos sur la jambe de sa mère qu'il a pu jouer. Plus tard, il lui faudra remplir complètement sa cavité buccale avec n'importe quel jouet pour pouvoir échanger avec moi dans un miroir.

Cela correspond aux observations d'Esther Bick (1968):

Le besoin d'un objet contenant, dans les états de non-intégration de la petite enfance, tend à produire une recherche frénétique pour un objet - une lumière, une voix, une odeur, un objet sensuel - qui puisse retenir l'attention et faire fonction de tenir ensemble les parties de la personnalité. Cet objet contenant est expérimenté concrètement comme une peau.

Les sensations corporelles non seulement tactiles, mais sonores, visuelles et d'équilibre, sont gardées en mémoire comme des représentations qui ne peuvent être mises en mots, ce que D. Anzieu a nommé les signifiants formels. Ils sont une forme dynamique et spatiale des contenants psychiques et 
se manifestent quand ils sont la trace d'un traumatisme infantile: «ça fait un trou, la peau rétrécit».

Les petits communiquent leurs sensations à la mère qui, si tout va bien, les traduit en affects et permet que le chaos et la désorganisation n'aient pas lieu.

Ceux qui ont pratiqué l'observation de nourrissons à domicile connaissent ces moments de malaise chez l'adulte qui regarde un nourrisson dans le bain, mal soutenu, avec l'impression soudaine de tomber et d'avoir un vertige à la limite de la nausée.

Un petit de garçon de 4 ans, dont la mère borderline ne pouvait le porter sans s'effondrer en larmes, m'a parlé en séance de la certitude qu'il avait que son corps allait fondre dans l'eau comme du sucre en poudre ou perdre sa forme pour toujours. Il me confia cette impression alors qu'il dessinait des animaux dont la peau devait être plusieurs fois entourée de nombreuses lignes et d'enveloppes successives, «sinon, me dit-il, tu vois, on s'écroule et la peau s'efface, alors on est liquide et ça tombe, ça fuit de partout et on est plus là, c'est mort.»

C'est aussi la fonction de l'analyste, avec son attention, sa préoccupation et ses interventions, de maintenir l'expérience du patient d'être tenu et contenu. Évelyne Séchaud disait «la psychanalyse est une peau vivante pour les pensées.»

Les analystes d'enfants connaissent bien ces patients aux symptômes somatiques; tics, hyperactivité, maladresses, associés avec des effrois autour de l'abandon et de la séparation. Ils rapportent combien la petite enfance était pleine de symptômes comme l'insomnie, les activités autocalmantes et le balancement répétitif. C’est Kernberg qui a rappelé que les faiblesses du Moi étaient liées à des difficultés à établir la constance de l'objet, ce qui rend alors l'enfant dépendant de la présence réelle de la mère. L'association d'activités autocalmantes et des limites à pouvoir être seul manifestent le déficit de capacité pour l'auto-érotisme et l'intégration des qualités libidinales du Moi-peau dans la relation avec l'objet maternel.

Garder les traces et recréer les expériences de plaisir sensuel avec la mère est une des meilleures façons de construire des représentations qui vont ensuite permettre à la capacité de rêverie et au fonctionnement transitionnel de se développer, donnant accès à une séparation d'avec l'objet sans perte de l'attachement.

Les défauts de cette étape se voient dans les formations de «seconde peau» observées par Esther Bick (1968). Protection contre une fonction 
maternelle défaillante, ces manifestations de défense rigide du self montrent aussi combien le processus de subjectivité est en difficulté. E. Bick décrit l'enfant «sac de pommes», facilement blessé, sans forme, en recherche constante d'attention et d'appréciation, toujours au bord de l'effondrement. L'autre cas est celui de la formation «d'hippopotame», où l'enfant est fermé au monde, agressif, tyrannique, toujours en insécurité et vite persécuté.

Ces formations précoces défensives protègent une très fragile intégration interne avec un défaut grave d'organisation de la continuité corporelle. Dans de tels cas, un symptôme précoce de troubles alimentaires a souvent été négligé par l'entourage.

Helen est une petite fille de 3 ans renvoyée de l'école maternelle du fait d'un cri intolérable au départ de sa mère, hurlement strident qui pouvait durer une heure. Elle mordait les enfants et léchait les chaussures des maitresses, alors qu'avec sa mère, collée à son corps, elle était une enfant harmonieuse et séductrice. Après plusieurs séances mère-enfant, je décide de demander à la mère de rester dans la salle d'attente, de nous donner son foulard et un objet que Helen choisit, et j'affronte le cri strident pour aller seule avec Helen dans mon bureau. Je lui dis qu'elle a perdu une partie de son corps en laissant maman seule au loin. Elle s'arrête de crier, et joue à tomber par terre, molle, sans forme. Je reprends ce «jeu» avec une poupée qui tombe de mes genoux et que je tente d'habiller et de tenir droite. Helen va investir ce jeu avec des ficelles qui vont tout d'abord tenir la poupée contre moi et qui ensuite, pendant des semaines de séances continues, serviront à nous attacher toutes les deux ensemble. Elle disait alors que cela protégeait du vertige de tomber.

Les épisodes de dissociation se sont apaisés et, quand elle a pu dessiner, nous avons fait des squiggles de ronds se contenant les uns les autres, tandis qu'elle criait "c'est Moi!», se sentant à la fois contenue et unifiée, dans un moment maniaque de sentiment narcissique d'exister avec une identité.

Plus tard, quand la sexualité génitale est en place, les fantasmes sont encore associés à la qualité de la relation d'objet. C'est ainsi que l'effet d'après-coup donne une valeur sexuelle aux évènements de la petite enfance, et que les déficits narcissiques de cette période laissent l'adulte avec un sentiment de vide et d'absurdité. L'importance des comportements d'addiction me semble être un reflet de ces problèmes, qui sont aigus dans notre société à l'heure actuelle.

Un patient adulte criait en séance son irritation au contact des autres. Intolérable, il disait se sentir «comme un crabe sans coquille, vide et sans 
vie», mais être envahi d'excitations pénibles au contact des autres. Enfant, il avait été soumis à l'hypersexualisation de la vie familiale, qui était sans filtre.

L'objet du premier attachement n'est pas seulement essentiel par sa qualité de limite contenante, c'est aussi un objet excitant, source d'investissement libidinal. Quand ensuite les parents d'un petit enfant qui marche et touche à tout interdisent le toucher, ils marquent un des premiers interdits œedipiens, comme l'a montré D. Anzieu. L'interdit du toucher demande la répression des pulsions et la capacité de se contenir, organisant aussi la faculté de représentation de la satisfaction.

L'usage du concept de Moi-peau, associé avec les apports de Winnicott et de Bion, permet de trouver des techniques d'intervention spécifiques pour chaque couple mère-enfant afin de les aider à réparer leurs mésententes. La notion de neuroplasticité et les liens entre le Moi-peau et la régulation psychosomatique manifestent combien ces interventions précoces sont efficaces sur les symptômes de l'enfant, et sans doute dans la prévention de troubles narcissiques.

Je vais présenter un cas de petit enfant avec sa famille qui montre la place des déficits précoces dans une dyade, le besoin de contenance et la carapace caractérielle qui peut se mettre en place pour pallier les manques narcissiques.

Auparavant, pour en terminer - si c'est possible - avec la transmission familiale, je voudrais ajouter que le fantasme d'exister à l'intérieur de la peau de quelqu'un d'autre est un fantasme fréquent chez les enfants de remplacement.

Ma grand-mère paternelle, Marguerite Anzieu, a commencé sa vie comme enfant de remplacement d'une petite fille morte dont la peau avait été brûlée vive, et qui s'appelait elle-même Marguerite. Mon père, Didier Anzieu, est né après le décès à la naissance d'une fille qui n'a pas été nommée; évènements qui ont déclenché chez ma grande-mère une réaction persécutive et le développement d'une paranoïa. Elle fut hospitalisée des années plus tard, en 1931, à l'hôpital Sainte-Anne à Paris, après un passage à l'acte criminel, et elle rencontra Lacan qui en fit son sujet de thèse.

Quand il eut 60 ans, D. Anzieu écrivit:

Je suis devenu psychanalyste pour soigner ma mère. Pas tant pour la soigner dans la réalité, même si je suis arrivé à m'occuper d'elle de façon heureuse dans la dernière partie de sa vie. Ce que je veux dire, c'est soigner ma mère en moi et chez les autres. Traiter, à travers les autres, cette mère 
menaçante et menacée... La capacité de penser aspire à une logique universelle utopique; elle est toujours interrompue par des impulsions psychiques et des échecs. Mais c'est un moment émouvant et essentiel pour un psychanalyste quand un patient accède à la possibilité de se penser par lui-même et de savoir penser les autres.

La transmission familiale est quand même moins toxique quand les parents ont fait une analyse!

\section{Dépression primaire, le rôle du jeu}

Qu'elle soit due aux turbulences émotionnelles du post-partum qui dépriment la mère, aux troubles de l'investissement parental ou au tempérament d'un bébé plus ou moins difficile à satisfaire ou à calmer, la blessure narcissique ressentie par l'enfant constitue une menace de dépression primaire.

Les angoisses dépressives sont fréquentes et passagères chez les bébés, comme les observations de nourrissons à leur domicile le montrent bien. Elles sont en général réparées par la qualité du holding psychique et physique. Mais un défaut de cette expérience, pour des raisons propres à la mère ou au bébé, contribue aux réactions de repli, aux désordres psychosomatiques et aux réactions négatives de l'enfant au sourire et au contact visuel.

Dans les tout premiers mois de la vie, l'enfant ne souffre pas de la perte d'un objet, la relation étant encore trop fragile. Mais il est dans la lente différenciation entre soi et l'objet, et c'est la qualité de l'investissement de l'objet, encore précaire, qui sera alors touchée. C’est pourquoi la fréquence des séances est essentielle pour maintenir la construction d'un lien fiable. Lorsque la dépression dite anaclitique n'apporte pas assez d'activité mentale à l'enfant, les jeux autoérotiques cessent, le fonctionnement devient opératoire; on le voit sur le visage de ces bébés sérieux, jamais déridés, sans tonus vital, pris souvent dans la répétition infernale des comportements autocalmants, comme Pauline.

Bowlby et Winnicott l'ont pointé chacun à leur façon, ce n'est pas la séparation qui entraine la dépression du bébé, c'est la perte de l'espoir. Un objet disparu autant qu'inaccessible, lors d'un sevrage trop rapide par exemple, une interaction avec une mère inanimée, sans vocalisations ni regards, une maladie ou encore une intervention chirurgicale, tout ceci peut supprimer les liens avec l'objet et laisser le corps et le psychisme démunis. 
Le nourrisson est atone, en retrait, ralenti, rapidement désorganisé sur le plan psychosomatique, et peut présenter les défenses pathologiques décrites par Selma Fraiberg, à savoir l'évitement du contact visuel, la paralysie du comportement, ou freezing, la transformation des affects quand, au lieu de manifester de la détresse ou de l'angoisse, un bébé maltraité manifeste une joie bruyante et une excitation désorganisée avec de l'autoagressivité (Fraiberg, 1982, p. 630).

Devant le danger de désobjectalisation, offrir une enveloppe psychique et physique, ferme et souple, est essentiel; ce serait un contenant introjectable pour le bébé, à condition que la rythmicité de l'accordage au moment des soins permette un vrai rassemblement des sens, une action transformatrice du flux pulsionnel comme le propose Bullinger (1998). C'est dire l'importance des interventions directes auprès des nourrissons dont les mères interagissent de façon inadéquate. Le processus thérapeutique modifie l'économie psychique, le jeu du thérapeute sollicite et mobilise l'intérêt du bébé, maintient son corps en action et suscite le déroulement du fantasme qui s'y associe.

Les grands modèles de jeu s'organisent d'ailleurs autour de l'absence: le fort da, le cache-cache, sont des jeux de la perte maitrisée. La découverte et la création de jeux et de plaisirs de tous registres - la resexualisation de la vie - manifestent toujours une amélioration chez un bébé; l'apparition du sourire chez les nourrissons impassibles et du rire chez les petits devenus enfin enthousiastes est un signe fiable de changement.

Le jeu, dans les thérapies précoces, remplit quantité de fonctions, entre la remise en jeu des capacités relationnelles de la mère, l'engagement dans une communication œil à œil, la sonorité bien rythmée avec un bébé qui n'attend que ça, ou la lente réanimation d'un nourrisson en retrait, hypotonique, presque sans désir. Autant de jeux d'imitation, d'accordage, de miroir sonore et visuel, offrent empathie, sympathie et capacité de lire les affects.

L'échange émotionnel est le lien entre le monde interne et l'extérieur, le matériau qui permet au lien de se créer et de se poursuivre. Le danger est moins la haine que le retrait, c'est-à-dire la non-émotion, ce que Bion appelle l'anti-émotion.

Permettre à un bébé d'agir sur le monde, de se sentir capable d'action sur son corps propre et sur les autres, est essentiel. Le jeu se déploie avec un plaisir partagé, la complicité amusée du thérapeute. Il est important de rester assez naïf et enthousiaste pour être surpris par le bébé et ses formidables capacités de relation et de récupération. 
Pouvoir intéresser un enfant et ses parents à son fonctionnement psychique est parfois difficile, mais c'est un moyen essentiel pour composer avec les exigences pulsionnelles. Pour ce faire, l'enfant doit pouvoir disposer d'un théâtre interne, lieu privilégié où il va pouvoir jouer avec ses objets internes lorsque la réalité ne répond pas à ses attentes. La technique psychanalytique et le cadre de nos traitements seront différents selon qu'il s'agira de construire le théâtre ou de transformer des scénarios dont la fixité tend à enfermer le sujet dans des impasses. Un des ressorts de cette entreprise réside dans le plaisir de fonctionnement partagé avec l'enfant.

Si l'objet maternel déçoit les attentes et ne permet pas de maintenir une continuité d'investissement, le déplaisir et l'expérience de la douleur désorganisent les premières représentations objectales. La décharge par le corps sera une solution rapide aux tensions.

Le jeu permet de figurer la réponse de l'objet, à savoir ce qui a été refusé ou a été impossible, les interdits comme les pertes. Le jeu permet aussi à l'enfant de satisfaire son besoin d'action et de transformation. Le thérapeute doit essayer de rester créatif pour jouer, de garder le sens de l'humour, puisque cela permet de maintenir la différenciation entre la réalité interne et la réalité externe, tout en jouant «pour de vrai» ou «pour de faux ». On reste dans le domaine de l'illusion, pour amener à des possibilités de représentation chez le bébé et pour lui permettre de s'approprier les processus de symbolisation.

\section{Capacité de rêverie et transitionnalité}

André Green a décrit le syndrome de désertification psychique des patients qui ne peuvent supporter le cadre et qui ressentent une vacuité angoissante. L'unité du moi est alors en danger, et le face-à-face offrant le regard et la présence physique de l'analyste permet au moi de surmonter la détresse. Les atteintes précoces du moi, leurs conséquences économiques autant que structurales, imposent des changements dans le travail analytique. Le trouble est compris comme la conséquence de la qualité de l'environnement primaire et le soin est pensé en accord: le cadre et la personne de l'analyste recomposent un environnement qui doit fournir des qualités telles que le transfert fera revivre un reflet des relations primitives.

Appuyé sur la rêverie de l'analyste et son attention à la destructivité comprise comme un enjeu du négatif, le patient rencontre un écho à sa souffrance mais aussi une limite à ses attaques: les expériences précoces d'attente vaine de l'objet et leur cortège de réactions destructives de la pensée sont 
associées aux failles de l'analyste et reprises dans la tiercéité. Remettre en route des mouvements libidinaux est lutter contre le vide. En ce sens, Green rappelle l'influence de la relation maternelle fusionnelle sur la libido «plus libre que liée».

La transitionnalité est essentielle, jouant sur le paradoxe d'être et de n'être pas, du semblable et du différent. L'espace transitionnel doit être continuellement recréé, et si l'espace de jeu est rétréci par la perte de confiance dans l'imago maternelle, l'analyse doit être le «pont jeté vers le monde» qui réanime l'espace potentiel de créativité (Schacht, 2001).

La créativité de l'analyste est essentielle dans ces cas difficiles, en ce qu'elle permet le dégagement de la pulsion et l'apaisement des enjeux de perte. Fonction d'intrication des pulsions, de la destructivité, le holding chez l'analyste permet le passage du sensoriel à l'activité de représentation. Être en empathie, faire sien l'autre, construire la structure encadrante, mais aussi jouer avec la perte et l'absence font travailler la bisexualité de l'analyste, en créant des liens et en manifestant une présence étayante, mais loin de la toute-puissance.

Les enfants en détresse comme les patients état limite manifestent l'intensité des atteintes précoces du narcissisme. L'importance de la relation à l'objet primaire maternel se rejoue dans le transfert, et la temporalité de l'après-coup permet parfois la reprise d'un processus de symbolisation.

\section{Pour conclure}

La joie de vivre est certes le premier stade de la libido retrouvée, mais il faut encore travailler à mettre en place les possibilités de symbolisation.

Tous les bébés qui rient ne sont pas guéris, mais ils sont sur la voie de la vitalité qui donne sa valeur à la libido pour affronter la position dépressive et les conflits odipiens, quand le rire s'associe au plaisir du corps et au jeu des représentations.

Didier Anzieu était connu pour son sens de l'humour, né sans doute des déceptions et douleurs de la vie qu'il avait dû gérer seul. Mais il a communiqué à ses élèves et ses enfants le grand plaisir de savoir en rire et de communiquer cet élan vital aux patients.

Dans la culture Navajo, l'enfant est un cadeau apporté par la nature que l'on doit respecter. L'évènement qui donne à l'enfant son nom et sa place dans la société est la cérémonie du Premier Rire. Cela assure à l'enfant d'être constamment sous l'attention d'un adulte, protégé dans son berceau et jamais laissé seul jusqu'à ce qu'il rie pour la première fois. C'est ce jour qui 
marque la naissance de l'enfant comme être social et une cérémonie spéciale marque ce premier rire.

Un vrai rire joyeux serait donc le signal de la solidité du moi.

\author{
Christine Anzieu-Premmereur \\ canzieu@gmail.com
}

\title{
Notes
}

1. Je veux remercier Sophie Gilbert et la revue Filigrane pour son soutien et son amitié, et pour m'avoir invitée à venir vous parler aujourd'hui.

\section{Références}

Anzieu, D. (1974). Le Moi-Peau. Nouvelle Revue de psychanalyse, 9, 195-208.

Anzieu, D. (1979). La démarche de l'analyse transitionnelle en psychanalyse individuelle.

Dans R. Kaës (dir.), Crise, rupture et dépassement (p. 184-219). Paris: Dunod.

Anzieu, D. (1985). Le Moi-Peau. Paris: Bordas. Trad. angl.: Anzieu, D. (1989). The Skin Ego: A

Psychoanalytic Approach to the Self. New Haven: Yale University Press.

Anzieu, D. (1986). Une peau pour les pensées. Paris: Clancier-Guenaud.

Anzieu, D. (1994). Le penser. Paris: Dunod.

Anzieu, D. et al. (1987). Les enveloppes psychiques. Paris: Dunod.

Anzieu-Premmereur, C. (2000). Le jeu dans la psychothérapie de l'enfant de 0 à 5 ans. Dans

A. Anzieu, C. Anzieu-Premmereur et S. Daymas (dir.), Le jeu en psychothérapie de l'enfant (p. 39-90). Paris: Dunod.

Anzieu-Premmereur, C. (2003). Illusion et jeu: la mise en place de l'espace transitionnel.

Dans C. Anzieu-Premmereur et M. Cornillot (dir.), Les pratiques psychanalytiques auprès des bébés (p. 19-56). Paris: Dunod.

Anzieu-Premmereur, C. (2011). Fondements maternels de la vie psychique. Revue française de psychanalyse, 75 (5), 1449-1488.

Bick, E. (1968). L'expérience de la peau dans les relations objectales précoces. Revue belge de psychanalyse, 50 (2007), 137-141.

Bion, W. (1962). Aux sources de l'expérience. Paris: Presses universitaires de France.

Bowlby, J. (2002). Attachement et perte: Vol. 1. L'attachement. Paris: Presses universitaires de France.

Bullinger, A. (1998). Le dialogue sensorimoteur avec l'enfant: les particularités du bébé à risque autistique. Dans P. Delion (dir.), Les bébés à risque autistique (p. 47-60). Paris: Eres.

Fraiberg, S. (1982). Pathological defenses in infancy. The Psychoanalytic Quarterly, 51 (4), 612-635.

Freud, S. (1915). Pulsions et destin des pulsions. Dans S. Freud (1976), Métapsychologie (p. 11-44). Paris: Gallimard.

Freud, S. (1923). Le Moi et le Ça. Dans S. Freud (1991), Euvres complètes. vol. XVI. 1921-1923 (p. 255-301). Paris: Presses universitaires de France.

Klein, M. (1952). Quelques conclusions théoriques au sujet de la vie émotionnelle des bébés. Dans M. Klein et al. (1980), Développements de la psychanalyse (p. 187-222). Paris: Presses universitaires de France.

Milner, M. (1976). L'inconscient et la peinture. Paris: Presses universitaires de France. 
Roussillon, R. (1995). La métapsychologie des processus et la transitionnalité. Revue française de psychanalyse, 59 (numéro spécial), 1349-1519.

Roussillon, R. (2000). Traumatismes primaires et liaisons non symboliques. Actualités psychosomatiques, 3, 89-109.

Schacht, L. (2001). La capacité d'être surpris. Journal de la psychanalyse d'enfant, 29, 195-214.

Winnicott, D. W. (1941). L'observation des jeunes enfants dans une situation établie. Dans D. W. Winnicott (1989, $2^{\mathrm{e}}$ éd.), De la pédiatrie à la psychanalyse (p. 269-288). Paris: Payot.

Winnicott, D. W. (1947). La haine dans le contre-transfert. Dans D. W. Winnicott (1989, $2^{\mathrm{e}}$ éd.), De la pédiatrie à la psychanalyse (p. 48-58). Paris: Payot.

Winnicott, D. W. (1951). Objets transitionnels et phénomènes transitionnels. Dans D. W. Winnicott (1989, $2^{\mathrm{e}}$ éd.), De la pédiatrie à la psychanalyse (p. 109-125). Paris: Payot.

Winnicott, D. W. (1968). L'utilisation de l'objet et le mode de relation à l'objet au travers des identifications. Dans D. W. Winnicott (1971), Jeu et réalité (p. 120-130). Paris: Gallimard. 